\title{
BMJ Open Qualitative approach to patient-reported outcomes in oncology: protocol of a French study
}

\author{
Massimiliano Orri, ${ }^{1,2,3}$ Jordan Sibeoni, ${ }^{1,2,4}$ Mathilde Labey, ${ }^{1,2}$ Guilhem Bousquet, ${ }^{5,6}$ \\ Laurence Verneuil, ${ }^{7,8}$ Anne Revah-Levy, ${ }^{1,2,9}$ QualiPRO study group
}

To cite: Orri M, Sibeoni J, Labey M, et al. Qualitative approach to patient-reported outcomes in oncology: protocol of a French study. BMJ Open 2015;5:e008042. doi:10.1136/bmjopen-2015008042

- Prepublication history for To view these files please visit the journal online (http://dx.doi.org/10.1136/ bmjopen-2015-008042).

Received 25 February 2015 Revised 20 April 2015 Accepted 23 April 2015 this paper is available online.

\section{ABSTRACT}

Introduction: The past decade has been characterised by movement from a doctor-centred to a patientcentred approach to treatment outcomes, in which doctors try to see the illness through their patients' eyes. Patients, family members and doctors are the three participants in cancer care, but their perspectives about what have been helpful during cancer treatment have never simultaneously and explicitly compared in the same qualitative study. The aim of this study project is to explore patients' perspectives about the care they receive, as well as families' and doctors' perspectives about what have been helpful for the patient. These three points of view will be compared and contrasted in order to analyse the convergences and divergences in these perspectives.

Methods and analysis: This is a national multicentre qualitative study. Participants will be constituted by three different subsamples: (1) patients with cancer (skin, breast, urological and lung cancers), (2) their relatives, and (3) their referring physicians.

Recruitment will follow the purposive sample technique, and the final sample size will be determined by data saturation. Data will be collected through openended semistructured interviews and independently analysed with NVivo V.10 software by three researchers according to the principles of Interpretative Phenomenological Analysis.

Ethics and dissemination: The research protocol received approval from the University Paris Descartes review board (IRB number: 20140600001072), and participants will provide written consent. To the best of our knowledge, this is the first study to focus on the simultaneous exploration of the separate points of view of patients, families and doctors about the care received during the cancer care journey. We expect that our findings will help to improve communication and relationships between doctors, patients and families. Comparison of these three points of view will provide information about the convergences and divergences of these perspectives and how to address the needs of all three groups.

For numbered affiliations see end of article.

Correspondence to

Massimiliano Orri;

massimiliano.orri@gmail.com

\section{INTRODUCTION} the care they receive during their cancer treatment are considered increasingly
Patients' own perspectives and perceptions of

\section{Strengths and limitations of this study}

- This study has been methodologically designed to ensure validity of our findings, as ensured by methodological accuracies such as the use of purposive sampling, data saturation and triangulation. Additionally, we will select patients affected by four cancer types (skin, urogenital, lung and breast) which differ in prognosis, treatment and clinical manifestations in order to enhance transferability of the findings.

- Potential limitations are: first, the results of our study can be transferred to other healthcare contexts only with caution, because cancer care depends strongly on medical system policies, as well as the economics of the country. Second, nurses' perspectives are lacking in this study. We made the choice to concentrate only on doctors for feasibility reasons, although we are aware that nurses are often the healthcare professionals with the most patient contact.

- Sample size will be adequate to ensure sufficient data concerning these four different cancer experiences (and avoid loss of precision).

important today. ${ }^{1}$ Accordingly, the third French Cancer Plan 2014-2018 states that "a quality relationship between patients and their healthcare providers is a condition for both care and communication that meet patients' expectations" (p.70). ${ }^{2}$

Advances in modern medicine and the development of evidence-based medicine (EBM) have made possible dramatic progress in oncology, in terms of survival, quality of care and availability of treatment. At the same time, the doctor-patient relationship has also changed almost as dramatically. ${ }^{3}$ Patients' preferences, choices and needs have been placed at the core of the decision-making process, because patients' feelings influence therapeutic choices, patient satisfaction and quality of life during and after the treatment period. ${ }^{3-6}$

Accordingly, the past decade has been characterised by movement from a doctor- 
centred to a patient-centred approach, in which doctors try to see the illness through their patients' eyes. ${ }^{78}$ This shift in the patient's role in care requires medicine to move beyond its traditional biomedical model and paternalistic approach (in which expert doctors based their decisions solely on diagnosis and pathophysiology), to take the patient's subjectivity into account. ${ }^{4}$ This patientoriented approach should be able to capture the dual dimension of every medical act: the care and the cure. $^{79}$

This new context has led to the emergence of patientreported outcomes (PROs), which can be defined as "any reports coming directly from patients about how they function or feel in relation to a health condition and its therapy." ${ }^{10}$ PROs provide patients' perspectives on treatment benefits and outcomes beyond survival, disease and physiological markers: they are often the outcomes of greatest importance to patients. PROs are elicited by methods such as interviews, self-completed questionnaires, diaries and other data collection tools, preferably specific methods that are rigorous, scientific and validated. ${ }^{10}$

The interest in patients' subjective perspectives has led researchers to recommend the use of interpretative research methods that can directly explore their point of view. ${ }^{11}$ Qualitative methods are the gold standard for research seeking to understand in depth complex phenomena from the perspective of the people directly involved. ${ }^{12}$ In the field of cancer care, qualitative methods have been successfully used to address topics such as barriers in help-seeking, ${ }^{13}$ doctor-patient communication ${ }^{14}$ and the needs of families and patients. ${ }^{15}$

This corpus of studies demonstrates the importance of psychosocial issues in the treatment of cancer; it also shows that families follow clear patterns of social, psychological and spiritual well-being and distress throughout the trajectory of their relative's illness. ${ }^{15}$ These patterns mirror the patients' experiences, but clinicians appear to be unaware of it-despite its quite negative effect on families' caregiving capacities. Moreover, other studies have shown that oncologists act according to what they think is best for the patient, trying to balance hope and uncertainty, but often resulting in collusion and false optimism. ${ }^{16}$ On the whole, the literature clearly shows a divergence between the perspectives of doctors, patients and families about cancer treatment-a divergence that leaves patients' needs substantially unaddressed.

Our project is primarily interested in examining the gap between these perspectives, by comparing the perspectives of patients, families and physicians about their representation of treatment. We have chosen an original approach that can deal directly with an issue only suggested by others-the goal of integrating these perspectives investigating simultaneously patients', families' and physicians' point of views about the same situation (ie, the cancer management of the patient). To the best of our knowledge, no study has yet attempted to achieve this explicit aim, and notable gaps in the literature remain unfilled.

\section{AIMS}

The aim of this study project is threefold:

1. To explore patients' perspective about the care they receive. We will address in particular their perceptions of what helped them during their treatment (in terms of both care and cure), what made them feel better able to handle their situation, and what made their illness harder for them;

2. To explore doctors' perceptions of what was helpful to patients;

3. To explore families' point of view about what was helpful during their loved one's cancer treatment.

These three points of view will be compared and contrasted to look for the features they share and those that differed in the representations of what was helpful during the treatment period and to analyse the convergences and divergences in these perspectives.

\section{RESEARCH TEAM}

The QualiPRO research team comprises both experienced qualitative researchers and clinicians working with people with cancer. The main investigators have backgrounds in psychiatry or psychology and substantial experience in conducting qualitative research (ARL, a psychiatrist, heads the qualitative research team within national research unit U669; JS is a special registrar in child psychiatry; ML is a psychiatrist-researcher; $\mathrm{MO}$ is a psychologist and $\mathrm{PhD}$ candidate). This team has already conducted several studies in various fields of adult and adolescent health (psychiatry, oncology, surgery and anaesthesiology). The relations between the perspectives of patients, family and healthcare providers have become the core topic of this research team, which is especially interested in shared representations of illness and care/treatment among the different stakeholders.

\section{METHODS}

Setting

This is a national multicentre study. Four departments are involved (3 in the Paris area: Paris Saint-Louis 1 and 2 and Bobigny-Avicenne; and one in Caen, in northern France; see figure 1 and table 1). All are teaching hospitals.

\section{Participant selection and recruitment}

The final sample will be constituted by three different subsamples: (1) patients with cancer, (2) their relatives, and (3) their referring physicians.

We have established a set of inclusion/exclusion criteria for the patients (box 1). Since a purposive sample technique ${ }^{17}$ will be used to obtain a maximum variation sample with a wide range of different experiences, we chose to concentrate on different kinds of cancer sites and different cancer stages (metastatic and nonmetastatic, specifically adult patients with cancer with the following diseases:

- Skin cancer: lymphoma, melanoma.

- Breast cancer. 


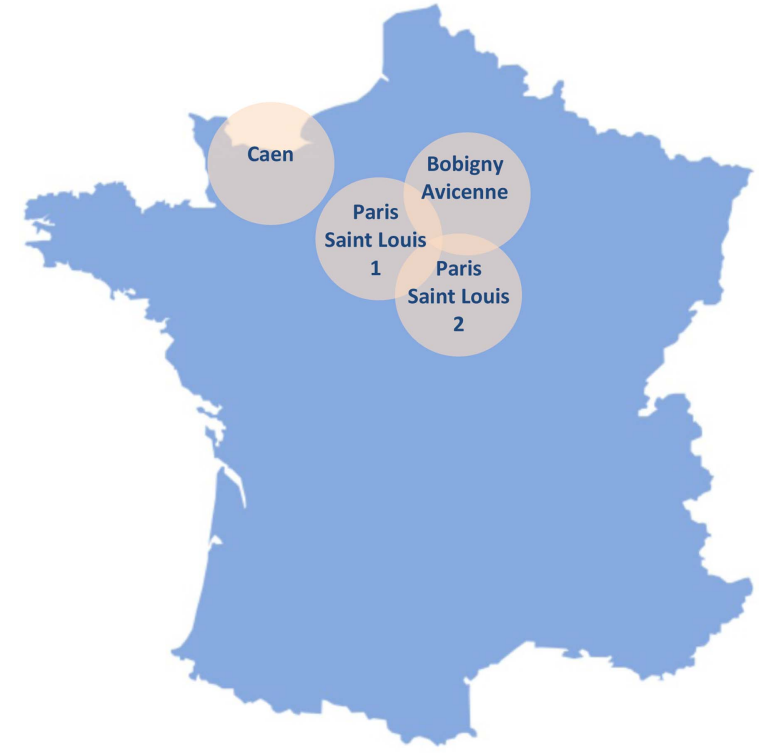

Figure 1 Geographical location of study sites. The figure shows the geographical location of the four centres participating in the study in the French territory.

- Kidney and urological cancers.

- Lung cancer.

A clinician coordinator has been identified for each participating centre. They will ask patients who meet the inclusion criteria (and their family members and physicians) to participate and seek to recruit both men and women in different age groups.

The size of the sample will be determined by data saturation, ${ }^{18}$ defined as the point when no new relevant information that increases our understanding of the phenomenon of interest emerges in the performed analysis. Analysis begins the month after data collection starts.

\section{Data collection}

Data will be collected through open-ended semistructured interviews with patients, a relative (spouse, sibling, child or parent) and a doctor directly involved in the patient's care. These interviews will elicit the representations of each of these groups about the actual care process.

The interviews will be conducted by experienced qualitative researchers, two men (MO and JS) and two women (ML and ARL), whose backgrounds have been
Box 1 Inclusion and exclusion criteria

Inclusion criteria

- Age: 18 years or older (no upper limit)

- Cancer site: breast, kidney, skin, urological

- In treatment for cancer for more than 6 months

- A close relative agrees to participate in the research

- Referring doctor agrees to participate in the research

- Able to communicate in French

Exclusion criteria

- Age: $<18$ years

- Cancer site other than the selected ones

- In the terminal phase (expected survival less than 6 months)

- No relative or referring doctor willing to participate in the research.

described above (see Research team section). A topic guide will be developed on the basis of a preliminary literature review and pilot interviews (with patient, family members and physicians) conducted by three different interviewers, analysed independently and discussed in a team (box 2).

A flexible topic guide-rather than a fixed schedulewas chosen because (1) our aim is to collect in-depth accounts, (2) the researchers all have substantial experience in conducting both open and semistructured interviews, and (3) we want to leave open the possibility of unpredicted issues that might be raised during the interviews.

The setting of these interviews will be the hospital in which the patients are treated. Researchers will meet the patient in a private room, provide all the explanations necessary, and obtain written informed consent. The interviews will last for about 30-60 min and be audiorecorded, and then transcribed verbatim and anonymised. Every nuance of the participants' narrative will be respected by transcribing pauses, silences and other non-verbal cues in the narratives.

\section{Data analysis}

A phenomenological framework will inform the data analysis. Phenomenology is the most suitable methodology for understanding how people subjectively perceive an important experience of their life and how they make sense of it. We have elected to perform a thematic content analysis according to the principles of Interpretative Phenomenological Analysis (IPA), which seeks to reach this understanding by adopting an

Table 1 Description of the study sites

\begin{tabular}{llll}
\hline Study site & Geographical location & Department & Cancer pathologies \\
\hline Paris Saint-Louis 1 & Paris-lle de France & Medical Oncology & Breast, lung, urogenital cancer \\
Paris Saint-Louis 2 & Paris-lle de France & Dermatology & Melanoma, skin lymphoma \\
Caen & Caen-Basse Normandie & Dermatology & Melanoma, skin lymphoma \\
Avicenne & Bobigny-lle de France & Radiation therapy & Breast, lung, urogenital cancer \\
\hline
\end{tabular}




\section{Box 2 Interview topic guide}

\section{Topic 1: Story of the illness}

Topic 2: Focus on the care received

- Pharmacological treatments (chemotherapy, radiotherapy, surgery)

- Complementary treatments (non-conventional treatments, psychosocial treatment, self-help group)

- Relationship with doctors/nurses

Topic 3: Coping with the emotional burden

'insider perspective'. ${ }^{19}$ IPA has three principal epistemological underpinnings. First, it is a phenomenological method that seeks to explore the participants' views of the world. According to Husserl, ${ }^{20}$ the objective of phenomenology is to understand how a phenomenon appears in the individual's conscious experience. Hence, experience is conceived as uniquely perspectival, embodied and situated. ${ }^{20}$ Second, IPA is based on interpretative activity (hermeneutics): Smith and Osborn ${ }^{19}$ define interpretation as a dual process in which the "researcher is trying to make sense of the participant trying to make sense of what is happening to them". During the analysis, the researcher might move dialectically between the whole and the parts, as well as between understanding and interpretation. Third, the idiographic approach emphasises a deep understanding of each case from the perspective and within the context of the individual. ${ }^{21}$

Five subsequent steps will follow

1. Three of the researchers, independently for all interviews, will begin by reading and rereading the entirety of each interview, to familiarise themselves with the participant's expressive style and to obtain an overall impression of the interview.

2. We will make initial notes, corresponding to the fundamental units of meaning. These notes will be descriptive and use the participant's own words; we will pay particular attention to linguistic details, such as the use of metaphors.

3. Conceptual notes will then be drafted, through processes of condensation, comparison and abstracting of the initial notes.

4. Connections with notes will be mapped and synthesised, and emergent themes developed. Each interview will be separately analysed in the same way.

5. Afterwards, the analysed interviews will be compared to enable us to cluster themes into categories and subcategories.

The independent analyses will be compared throughout the process to reach agreement. Every discrepancy will be negotiated within the research team and during regular meetings. NVivo V.10 $10^{22}$ will be used to manage the data and perform the analysis.

\section{Ensuring validity of the findings and methodological quality} Several procedures will be followed to ensure the validity and rigour of our findings. First, the purposive sample technique we are adopting (the best validated sampling method in qualitative research) ${ }^{1723}$ aims to select participants for their diversity rather than for their homogeneity. This ensures that stereotypical and common findings are challenged and enables us to describe the phenomenon under study in all its nuances. Second, the criteria of data saturation-rather than setting a fixed sample size-will enable us to stop inclusions only when the phenomenon we are investigating has been fully explored. Third, independent analysis by three researchers and the subsequent triangulation and discussion within the multidisciplinary research team will ensure the validity and inter-subjectivity of the analytic process. Fourth, we will pay careful attention to negative cases during the data analysis to integrate the participants' convergent but also divergent voices. Lastly, to ensure that our report meets high methodological standards, it will use the 32-item COREQ (consolidated criteria for reporting qualitative research) checklist. $^{24}$

\section{Reflexivity}

An important issue that we want to address is reflexivity, which can be defined as the reflection by the researchers of their role in the study and its effects on their findings at every step of the research process. ${ }^{25}$ To account for these influences, the researchers will share their preconceptions and make their positions clear during group meetings. We will also consider the emotional impact of the research subject on researchers themselves. For this reason, after each interview, researchers will complete a sheet (composed by 7 open questions, see box 3) about their own feelings and emotions during the interview. These sheets will be analysed and discussed during supervision sessions.

\section{Ethics}

Participants will receive complete written information about the scope of the research, the identity and affiliation of the researchers, the possibility of withdrawing from the study at any point, confidentiality and all other information required in accordance with French policies for biomedical research and with the Helsinki Declaration, as revised in 1989. Participants will provide

\section{Box 3 Researchers sheet}

Q1. Describe your emotions before the interview?

Q2. Describe your emotions during the interview?

Q3. What were you thinking during the interview?

Q4. Did you modify the way of conducting the interview according to what you were feeling? If yes, how?

Q5. Are there any topics that you regret having broached?

Q6. Are there any topic/aspect you did not investigate because of your feelings, even though you were supposed to, and if so, which?

Q7. Did you have any recurrent or embarrassing feelings which make you feel uncomfortable during the interview? 
Months

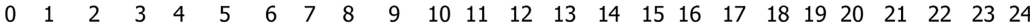

\begin{tabular}{|l|l|l|l|l|l|l|l|l|l|l|l|l|l|l|l|l|l|l|l|l|l|l|}
\hline $\begin{array}{l}\text { Initial meetings, coordination, } \\
\text { allocation of resources }\end{array}$ & & & & & & & & & & & & & & & & & & & & & & \\
\hline Pilot interviews & & & & & & & & & & & & & & & & & & & & & & \\
\hline Start recruitment and data collection & & & & & & & & & & & & & & & & & & & & & & \\
\hline Data analysis & & & & & & & & & & & & & & & & & & & & & & \\
\hline Publication and dissemination & & & & & & & & & & & & & & & & & & & & & & \\
\hline
\end{tabular}

Figure 2 Gantt diagram of study timescale. The figure represents how the five main phases of the study are organised in the 2-year project.

written consent. The research protocol received approval from the University Paris Descartes review board (Conseil d'évaluation éthique pour les recherchers en santé, CERES; IRB number: 20140600001072).

\section{TIMELINE}

This is a 2-year project. Figure 2 sets forth the planned timeline. After a 2-month coordination phase, study team organisational meetings, and allocation of resources, we will start three pilot interviews to verify the pertinence of our question guide. These interviews and their analysis and discussion will take 1 month. After the pilot phase, we will start data collection, which will take 7 months. Analysis will start almost simultaneously, a month after the interviews begin, and will be completed at month 18 after the beginning of the study. The remaining time will be dedicated to the diffusion of our results (drafting journal articles and conference presentations) and to the preparation of the final report for the funders.

\section{CONCLUSION}

To the best of our knowledge, this is the first study to focus on the simultaneous exploration of the separate points of view of patients, families and doctors about the care received during the cancer care journey.

We expect that our findings will help to improve communication and relationships between doctors, patients and families. Communication is the starting point for reaching a common representation of care, which is essential in meeting patients' needs in cancer care, especially within the medical model that emphasises shared decisionmaking and patient participation in choosing the treatment that best reflects their preferences and priorities. ${ }^{3}{ }^{4}$ Comparison of the perspectives of patients, their families and their doctors will provide information about the convergences and divergences of these perspectives and how to address the needs of all three groups; it should therefore help to promote their collaboration.

\section{Potential strengths and limitations}

This study has been methodologically designed to ensure validity of our findings, as discussed above. Additionally, we will select patients affected by four cancer types (skin, urogenital, lung and breast). Although this study will not address all types or sites of cancer, we believe that our findings will be transferrable to a large proportion of patients with cancer, because they are usually treated with a wide range of therapies (chemotherapy, surgery, hormone therapy and radiation therapy) and have a wide variety of prognoses (from melanoma with its high mortality rate to lymphoma and its low mortality).

Moreover, cancer care is a model of chronic disease and our findings may be transferred to other contexts such as severe diabetes care.

However, we are aware of potential limitations. First, the results of our study can be transferred to other healthcare contexts only with caution, because cancer care depends strongly on medical system policies, as well as the economics of the country. Second, nurses' perspectives are lacking in this study. We made the choice to concentrate only on doctors for feasibility reasons, although we are aware that nurses are often the healthcare professionals with the most patient contact. Other studies will be conducted to address this point.

\section{Author affiliations}

${ }^{1}$ INSERM 1178 research unit, Paris, France

${ }^{2}$ Paris-Sud University and Paris-Descartes University, Paris, France

${ }^{3}$ Department of Psychology, CRP-CPO Lab, University of Picardie Jules Vernes, Amiens, France

${ }^{4}$ Maison de Solenn, AP-HP Cochin Hospital, Paris, France

${ }^{5}$ Department of Medical Oncology, AP-HP Saint-Louis Hospital, Paris, France

${ }^{6}$ Paris-Diderot University, Paris, France

${ }^{7}$ Department of Dermatology, CHRU Caen, Caen, France

${ }^{8} \mathrm{Caen}$ Basse Normandie University, Caen, France

${ }^{9}$ Centre de Soins Psychotherapeutiques de Transition pour Adolescents, Argenteuil Hospital Centre, Argenteuil, France

Acknowledgements The authors would like to thank Ms JoAnn Cahn for revision of the English.

Collaborators QualiPRO study group contributors: ARL, principal investigator; $\mathrm{MO}, \mathrm{JS}, \mathrm{ML}, \mathrm{GB}$ and LV, co-investigators.

Contributors M0, JS, ML, GB, LV and AR-L elaborated the study protocol. MO wrote the initial manuscript. MO, JS, ML, GB, LV and AR-L reviewed the initial version of the manuscript and approved the final version.

Funding This work was supported by the Fondation de France, grant "Soigner, soulager, accompagner" 2014 number 00050334. 
Competing interests None declared.

Ethics approval Conseil d'évaluation éthique pour les recherchers en santé (CERES), University Paris Descartes.

Provenance and peer review Not commissioned; peer reviewed for ethical and funding approval prior to submission.

Open Access This is an Open Access article distributed in accordance with the Creative Commons Attribution Non Commercial (CC BY-NC 4.0) license, which permits others to distribute, remix, adapt, build upon this work noncommercially, and license their derivative works on different terms, provided the original work is properly cited and the use is non-commercial. See: http:// creativecommons.org/licenses/by-nc/4.0/

\section{REFERENCES}

1. Deshpande PR, Rajan S, Sudeepthi BL, et al. Patient-reported outcomes: a new era in clinical research. Perspect Clin Res 2011;2:137-44.

2. Vernant JP. Recommandations pour le troisième Plan Cancer [Guidelines for the third Cancer Plan]. 2013:1-165. http://www.sante. gouv.fr/IMG/pdf/Recommandations-pour-le-3e-plan-cancer.pdf (accessed 3 Nov 2014)

3. Truog RD. Patients and doctors-evolution of a relationship. N Engl $J$ Med 2012;366:581-5.

4. Mulley AG, Trimble C, Elwyn G. Stop the silent misdiagnosis: patients' preferences matter. BMJ 2012;345:e6572.

5. Sitzia J, Wood N. Patient satisfaction: a review of issues and concepts. Soc Sci Med 1997;45:1829-43.

6. Sanda MG, Dunn RL, Michalski J, et al. Quality of life and satisfaction with outcome among prostate-cancer survivors. $N$ Engl $J$ Med 2008;358:1250-61.

7. De Valck C, Bensing J, Bruynooghe R, et al. Cure-oriented versus care-oriented attitudes in medicine. Patient Educ Couns 2001;45:119-26.

8. Mac Whitney J. Patient-centered and doctor-centered models of decision making. In: Sheldon M, Brook J, Rector A, eds. Decision making in general practice. Stockton; London, 1985.

9. Bensing J. Bridging the gap: the separate worlds of evidence-based medicine and patient-centered medicine. Patient Educ Couns 2000;39:17-25.
10. 17.1 What are patient-reported outcomes? http://handbook. cochrane.org/chapter_17/17_1_what_are_patient_reported outcomes.htm (accessed 26 Sep 2013).

11. Lewin S, Glenton C, Oxman AD. Use of qualitative methods alongside randomised controlled trials of complex healthcare interventions: methodological study. BMJ 2009;339:b3496.

12. Pope $\mathrm{C}$, Mays N. Reaching the parts other methods cannot reach: an introduction to qualitative methods in health and health services research. BMJ 1995;311:42-5.

13. Smith LK, Pope C, Botha JL. Patients' help-seeking experiences and delay in cancer presentation: a qualitative synthesis. Lancet 2005;366:825-31

14. The AM, Hak T, Koëter G, et al. Collusion in doctor-patient communication about imminent death: an ethnographic study. BMJ 2000;321:1376-81.

15. Murray SA, Kendall M, Boyd K, et al. Archetypal trajectories of social, psychological, and spiritual wellbeing and distress in family care givers of patients with lung cancer: secondary analysis of serial qualitative interviews. BMJ 2010;340:c2581.

16. Audrey S, Abel J, Blazeby JM, et al. What oncologists tell patients about survival benefits of palliative chemotherapy and implications for informed consent: qualitative study. BMJ 2008;337: a752.

17. Patton MQ. Qualitative research \& evaluation methods. 3rd edn. SAGE Publications, Inc, 2001.

18. Morse JM. The significance of saturation. Qual Health Res 1995; (5): $147-9$

19. Smith J, Osborn M. Interpretative phenomenological analysis. In: Smith JA, ed. Qualitative psychology: a practical guide to methods. Second edn. London: SAGE, 2008:53-80.

20. Husserl E. Cartesian meditations: an introduction to phenomenology. Martinus Nijhoff Pub, 1977.

21. Smith J, Flower P, Larkin M. Interpretative phenomenological analysis: theory, method and research. London: SAGE, 2009.

22. NVivo qualitative data analysis software; QSR International Pty Ltd Version 10, 2012.

23. Kuper A, Lingard L, Levinson W. Critically appraising qualitative research. BMJ 2008;337:a1035.

24. Tong A, Sainsbury P, Craig J. Consolidated criteria for reporting qualitative research (COREQ): a 32-item checklist for interviews and focus groups. Int J Qual Health Care 2007;19:349-57.

25. Malterud K. Qualitative research: standards, challenges, and guidelines. Lancet 2001;358:483-8. 